\title{
Factores relacionados con el control glucémico de pacientes con diabetes tipo 2
}

\author{
J. A. GIMENO ORNA, B. BONED JULIANI', L. M. LOU ARNAL, F. J. CASTRO \\ $\mathrm{ALONSO}^{2}$
}

Secciones de Medicina Interna, 'Bioquímica y ${ }^{2}$ Oftalmología. Hospital Comarcal de Alcañiz. Teruel

\author{
GLYCAEMIC CONTROL RELATED FACTORS IN TYPE 2 DIABETES \\ MELLITUS PATIENTS
}

\section{RESUMEN}

Objetivos: 1. Evaluar el grado de control glucémico, lipídico y de tensión arterial en una muestra de pacientes con diabetes tipo 2 .

2. Identificar factores predictivos del control glucémico promedio durante el seguimiento de los pacientes.

Material y métodos: Estudio de cohortes retrospectivo. Selección entre 2/1/00 y 14/11/01 de diabéticos tipo 2 visitados al menos 1 vez al año durante al menos 3 años en consultas externas hospitalarias. Clasificación en 3 grupos: a) tratamiento inicial y final con dieta y/o antidiabéticos orales; b) insulinizados durante el seguimiento; y c) tratamiento inicial y final con insulina. Determinación de índice de masa corporal (IMC), tensión arterial sistólica y diastólica, HbA1c y perfil lipídico inicial y final. Construcción de modelo de regresión múltiple con fines predictivos tomando como variable dependiente la $\mathrm{HbA} 1 \mathrm{c}$ media durante el seguimiento.

Resultados: Se seleccionaron 320 pacientes, 199 (62,2\%) mujeres y $121(37,8 \%)$ varones. Edad media 69 años (DE 9). Duración media del seguimiento de 5,2 años (DE 1,1). El grupo A estuvo constituido por 106 $(33,8 \%)$ pacientes, el grupo B por $70(22,4 \%)$ y el grupo C por 137 $(43,8 \%)$. Hubo un $63 \%$ de pacientes con $\mathrm{HbA} 1 \mathrm{c}>7,5 \%$, un $36 \%$ con tensión arterial > 140/85 mmHg y un $8,5 \%$ con LDL > $155 \mathrm{mg} / \mathrm{dl}$. La HbA1c media durante el seguimiento fue de $8,2 \%$ (DE 1,1 ) y sus predictores independientes: HbA1c inicial (0,37; IC 95\% 0,31-0,43; $\mathrm{p}<0,001)$, IMC inicial $(0,023$; IC 95\% 0,004-0,042; $\mathrm{p}=0,017)$, tratamiento inicial con insulina $(0,67$; IC $95 \% 0,45-0,88 ; p<0,001)$ y necesidad de insulinización (0,46; IC 95\% 0,21-0,71; p<0,001).

Conclusiones: Es difícil conseguir los objetivos de control glucémico recomendados. Los factores asociados a HbA1c media son la $\mathrm{HbA} 1 \mathrm{c}$ y el IMC iniciales y la necesidad de tratamiento insulínico.

PALABRAS CLAVE: Diabetes tipo 2. Control metabólico. Control glucémico.

\section{ABSTRACT}

Objectives: To describe metabolic, lipidic and blood pressure con trol and to identify blood glucose control predictive factors in patients with type 2 diabetes.

Material and methods: Retrospective cohort study. Ambulatory type 2 diabetic patients were selected from 2/1/00 to 14/11/01 if they had been visited yearly at least three years previously. They were classified into three groups: a) initial and final treatment with diet and/or oral glucoselowering drugs; $b$ ) insulinized patients during follow-up; and c) initial and final therapy with insulin.

Body mass index (BMI), blood pressure, HbAlc and lipid profile were determined at start and at the end of the study. A multiple regres sion model was used to predict mean HbAlc through follow-up.

Results: 320 patients were selected , 199 women $(62.2 \%)$ and 121 men (37.8\%) with mean age of 69 years (SD: 9). Mean follow-up was 5.2 years (SD: 1.1$)$.

106 patients $(33.8 \%)$ were included into A group, 70 patients (22.4\%) into B group and 137 (43.8\%) into C group. 63\% of patients had values of HbAlc > 7.5\%, $36 \%$ blood pressure > $140 / 85 \mathrm{mmHg}$ and $8.5 \%$ $L D L$ values $>155 \mathrm{mg} / \mathrm{dl}$.

HbAlc mean through follow-up was of $8.2 \%$ and its independent predictors were baseline HbAlc (0.37; IC 95\% 0.31-0.43; $p<0.001)$, baseline BMI (0.023; IC 95\% 0.004-0.042; $p=0.017)$, baseline treatment with insulin (0.67; IC 95\% 0.45-0.88; $p<0.001)$ and insulinization during follow-up (0.46; IC 95\% 0.21-0.71; $p<0.001)$.

Conclusions: It's difficult to achieve an acceptable blood glucose control. Mean HbAlc predictive factors include baseline HbAlc and $B M I$ and insulin therapy.

KEY WORDS: Type 2 diabetes. Metabolic control. Blood glucose con trol.

Gimeno Orna JA, Boned Juliani B, Lou Arnal LM, Castro Alonso FJ. Factores relacionados con el control glucémico de pacientes con diabe tes tipo 2. An Med Interna (Madrid) 2003; 20: 122-126.

\section{INTRODUCCIÓN}

La diabetes tipo 2 es una enfermedad de elevada prevalencia (1), con una considerable morbimortalidad asociada.

La importancia del control glucémico en la prevención de las complicaciones microvasculares ha sido confirmada en pacientes con diabetes tipo $1(2)$ y tipo $2(3,4)$. La medida de control glucémico en estos estudios ha sido el promedio de determinaciones de hemoglobina glicada (HbAlc) durante el seguimiento. La principal causa de mortalidad en la diabetes tipo 2 es cardiovascular (5). Para reducir la morbimortalidad cardiovascular se han mostrado eficaces el control lipídico (6)

Trabajo aceptado: 22 de noviembre de 2002

Correspondencia: Jose Antonio Gimeno Orna. Balbino Orensanz, 19, 2º B. 50014 Zaragoza. e-mail: mjpnosti@eresmas.com 
y el control de la tensión arterial (7), mientras que la eficacia del control glucémico es más controvertida (4).

Por ello las sociedades de diabetes Europea (8) y Americana (9) han recomendado la consecución de unos objetivos de control glucémico, lipídico y de tensión arterial cada vez más estrictos, con el objetivo de reducir las complicaciones crónicas micro y macroangiopáticas del paciente diabético. Específicamente, la guía del European Diabetes Policy Group (8), recomienda realizar controles de calidad periódicos para evaluar el grado de cumplimentación de los objetivos terapeúticos.

Los objetivos del presente trabajo fueron:

1. Evaluar el grado de control glucémico, lipídico y de tensión arterial en una muestra de pacientes con diabetes tipo 2 atendidos regularmente en consultas externas hospitalarias.

2. Comparar el control final con el que presentaban la primera vez que fueron atendidos en el centro hospitalario.

3. Identificar factores predictivos del control glucémico promedio durante el seguimiento de los pacientes.

\section{MATERIAL Y MÉTODOS}

Diseño: Para el primer objetivo se utilizó un diseño descriptivo transversal. Posteriormente se realizó un estudio de cohortes retrospectivo, mediante revisión de la historia clínica del paciente desde la primera vez que había sido visitado en nuestro centro.

Población a estudio: Se seleccionaron pacientes atendidos en consultas externas de endocrinología del Hospital Comarcal de Alcañiz (Teruel, España), con una población de referencia de 70.000 personas de un área rural.

Los criterios de inclusión fueron:

1. Diabetes tipo 2 diagnosticada por los criterios de la ADA (10).

2. Haber sido visitados regularmente en nuestro hospital (periodicidad mínima una vez por año) durante al menos 3 años.

Los pacientes se dividieron en 3 grupos: grupo A: pacientes en tratamiento inicial con dieta o antidiabéticos orales y tratamiento final con antidiabéticos orales; grupo B: pacientes en tratamiento inicial con dieta o antidiabéticos orales y tratamiento final con insulina (insulinización); grupo $\mathrm{C}$ : pacientes en tratamiento insulínico desde el principio.

Seguimiento: Se definió como duración del seguimiento el periodo comprendido entre la fecha inicial (primera visita en nuestro centro) y la fecha final (última visita recogida en nuestro centro). La primera visita se produjo entre el 1/6/94 y el 1/6/98; la última se realizó entre el 2/1/00 y el 14/11/01.

Variables analizadas: se determinó la edad, sexo y tiempo de evolución de la enfermedad en el momento de la visita final.

En la fecha inicial y final se determinaron variables clínicas y bioquímicas.

—Variables clínicas: peso en kg (medido en ropa ligera y sin zapatos con precisión de $500 \mathrm{~g}$ ), talla (precisión de $1 \mathrm{~cm}$ ), índice de masa corporal (IMC) definido como peso en $\mathrm{kg} / \mathrm{talla}$ al cuadrado en metros y tensión arterial sistólica y diastólica (promedio de tres determinaciones obtenidas tras 10 minutos de reposo en una posición sentada, con un manguito adecuado a la circunferencia del brazo y con una precisión de $5 \mathrm{mmHg}$ ).

- Variables bioquímicas: Se realizó extracción de muestra de sangre venosa tras ayuno nocturno de 10 horas y antes de la administración de la medicación antidiabética. Se analizaron glucemia, hemoglobina glicada (HbA1c), colesterol total, triglicéridos, y HDL colesterol.

Se definió el cambio de IMC en \% como (IMC final IMC inicial)/IMC inicial x 100.

Se definió la HbA1c media como el promedio de todas las determinaciones de hemoglobina glicada de cada paciente durante el seguimiento.

Métodos bioquímicos: Las mediciones de colesterol, triglicéridos, HDL y glucosa se realizaron mediante autoanalizador Shimadzu CL 7200. La HbA1c se midió en analizador automático Cobas Mira Plus, con un rango de normalidad entre 4,5 y $5,7 \%$. El LDL colesterol se calculó con la fórmula de Friedewald cuando los triglicéridos fueron inferiores a 400 $\mathrm{mg} / \mathrm{dl}$.

Métodos estadísticos: La descripción de variables cuantitativas se realizó mediante mediciones basadas en momentos (media y desviación estándar) y en ordenaciones (mediana y percentiles). La descripción de variables cualitativas se realizó mediante distribución de frecuencias (valoración del porcentaje de pacientes que cumplían los objetivos óptimos y aceptables de control glucémico, lipídico y tensional recomendados por el European Diabetes Policy Group).

La comparación de variables cuantitativas entre los momentos inicial y final se hizo mediante t de Student para datos pareados.

Se utilizó regresión simple y múltiple con fines predictivos. La variable dependiente fue la HbA1c media. Como variables independientes se introdujeron en los modelos: edad, sexo, tiempo de evolución, IMC inicial, cambio de IMC durante el seguimiento, HbA1c inicial, tratamiento inicial con insulina y necesidad de insulinización durante el seguimiento. Se realizó procedimiento de exclusión secuencial para seleccionar el mejor modelo estadístico.

$\mathrm{Se}$ condideraron significativas las asociaciones con $\mathrm{p}<0,05$. El programa estadístico utilizado fue SPSS 10.0.

\section{RESULTADOS}

Se seleccionaron 320 pacientes que cumplían los criterios de inclusión. De ellos 199 (62,2\%) eran mujeres y 121 $(37,8 \%)$ varones. La edad media fue de 69 años (DE 9; mediana 70,6). El tiempo de evolución fue de 16,5 años (DE 7,7; mediana 15,3)

La descripción del control glucémico, lipídico y de tensión arterial de los pacientes en la visita final quedan reflejados en la Tabla I.

La distribución del porcentaje de pacientes con consecución de los objetivos terapeúticos del European Diabetes Policy Group quedan reflejados en las Figuras 1 y 2 . Es de destacar que más del $50 \%$ de los pacientes conseguían un control óptimo de lípidos y de tensión arterial, pero apenas un $10 \%$ tenían una cifra ideal de HbA1c, definida como HbA1c < 6,5\%.

En la parte longitudinal del estudio la duración media del seguimiento fue de 5,2 años (DE 1,1; mediana 5,5). El número medio de determinaciones de HbA1c fue de 9,4 (DE 4,1; mediana 9). En la visita inicial había 9 pacientes $(2,8 \%)$ en tratamiento con dieta, $167(52,5 \%)$ en tratamiento con antidiabéticos orales y $142(44,7 \%)$ en tratamiento con insulina, mientras que en dos pacientes no se pudo determinar con seguridad. En la visita final había 111 
TABLA I

M EDIA, M EDIANA Y PERCENTILES DE HBA1C, TENSIÓ N ARTERIAL Y PARÁM ETROS LIPÍDICOS EN LA VISITA FINAL

\begin{tabular}{lccccccc}
\hline & MEDIA & DE & P 10 & P 25 & P 50 & P 75 & P 90 \\
\hline HbA1c (\%) & 8,1 & 1,4 & 6,5 & 7,2 & 7,9 & 8,8 & 9,9 \\
Colesterol (mg/dl) & 195 & 39 & 147 & 170 & 192 & 213 & 241 \\
Triglicéridos (mg/dl) & 140 & 94 & 67 & 83 & 111 & 164 & 252 \\
HDL (mg/dl) & 51 & 14 & 35 & 42 & 50 & 59 & 68 \\
LDL $(\mathrm{mg} / \mathrm{dl})$ & 116 & 29 & 81 & 97 & 113 & 133 & 153 \\
TAS $(\mathrm{mmHg})$ & 140 & 17,5 & 120 & 130 & 140 & 150 & 160 \\
TAD $(\mathrm{mmHg})$ & 76 & 9,8 & 60 & 70 & 75 & 80 & 90 \\
\hline
\end{tabular}
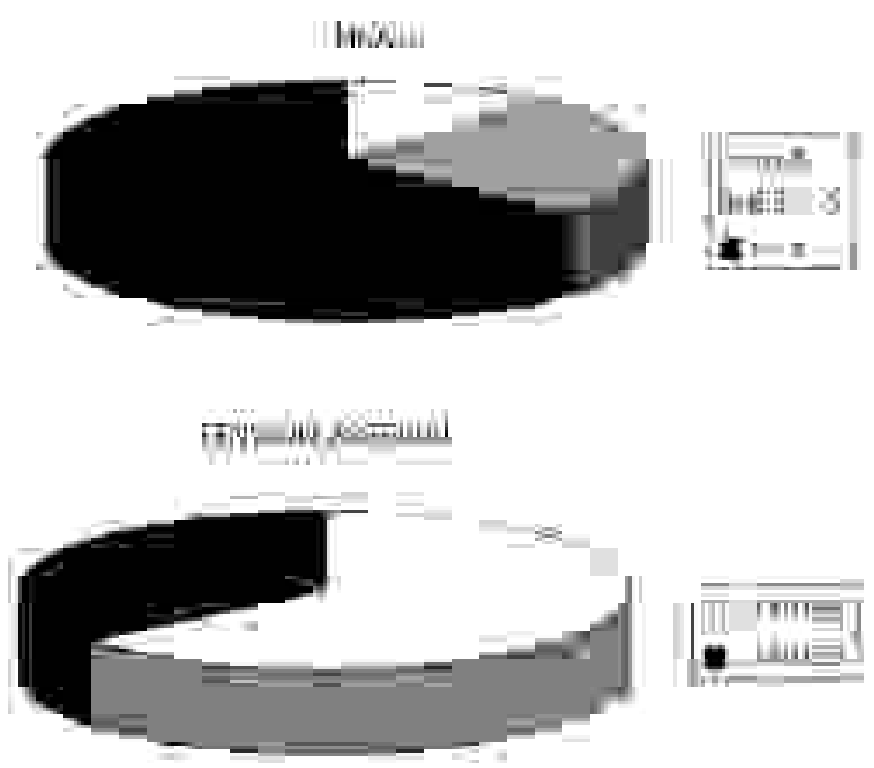

Fig. 1. Distribución de frecuencias de las cifras de $\mathrm{HbAlc}$ y de tensión arterial.
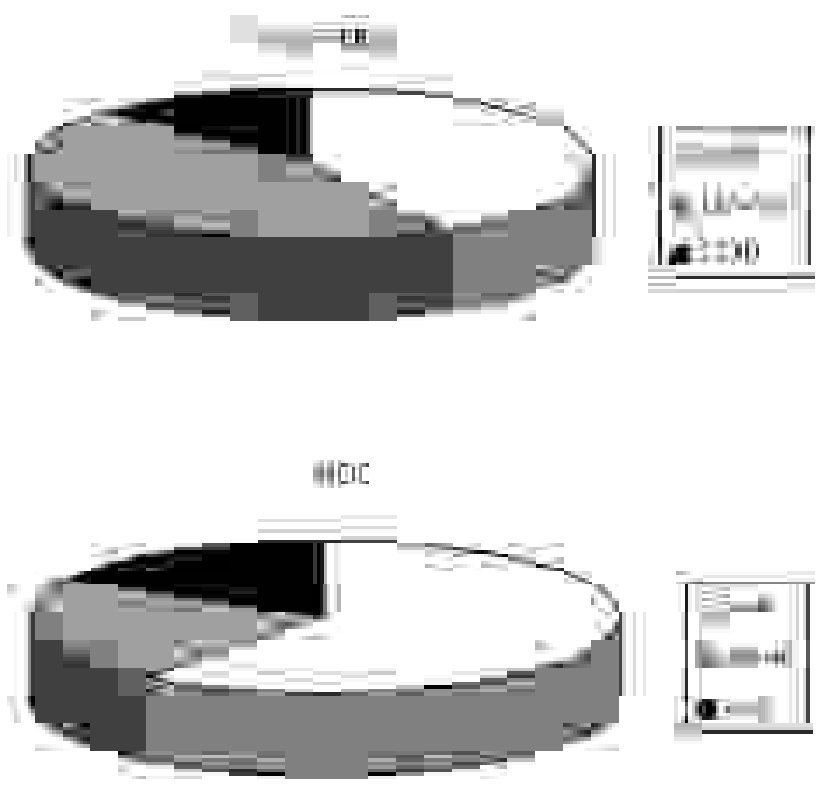

$(34,7 \%)$ pacientes en tratamiento con antidiabéticos orales y $209(65,3 \%)$ con insulina. Así el grupo A estuvo constituido por $106(33,8 \%)$ pacientes, el grupo B por $70(22,4 \%)$ y el grupo C por 137 (43,8\%); hubo 7 pacientes que no cumplieron criterios para ser incluidos en ninguno de los grupos.

Hubo diferencias significativas entre la visita inicial y la final en colesterol total (221 vs 195; $\mathrm{p}<0,001)$, HDL (43 vs $51 ; \mathrm{p}<0,001)$ y LDL (150 vs 115; p<0,001), tensión arterial sistólica (145 vs 140; $\mathrm{p}=0,001)$ y diastólica (79 vs $76 ; \mathrm{p}<0,001)$ e IMC $(29,3$ vs $30 ; \mathrm{p}<0,001)$. No hubo diferencias significativas en $\mathrm{HbA} 1 \mathrm{c}(7,9$ vs 8,06$)$ ni en triglicéridos (139 vs 140). Una descripción más detallada de la diferencia entre $\mathrm{HbA} 1 \mathrm{c}$ e IMC iniciales y finales en los grupos A, B, y C puede verse en la Figura 3. Llama la atención que sólo hubo empeoramiento significativo de HbA1c durante el seguimiento en el grupo A $(7,2$ vs 7,7 ; $\mathrm{p}=0,001)$, grupo en el que no hubo incremento de IMC $(28,9$ vs 28,8). Por el contrario en los grupos B y C no hubo empeoramiento significativo de $\mathrm{HbA} 1 \mathrm{c}$, pero sí incremento de IMC (grupo B: 30,2 vs 31,8; p<0,001; grupo C: 29,3 vs $30 ; \mathrm{p}<0,001)$.

La HbA1c media durante el seguimiento fue de $8,2 \%$ (DE 1,1 ; mediana 8,1).

Los modelos univariantes, utilizando como variable dependiente la HbA1c media durante el seguimiento, identificaron como variables significativas la $\mathrm{HbA} 1 \mathrm{c}$ inicial, el sexo masculino, el IMC inicial, el incremento de IMC durante el seguimiento, el tiempo de evolución, la presencia de tratamiento insulínico inicial y la necesidad de insulinización durante el seguimiento (Tabla II).

Tras regresión múltiple con procedimiento de exclusión secuencial el mejor modelo predictivo, con $\mathrm{R}$ cuadrado de 0,46 , fue el compuesto por 4 variables: HbA1c inicial $(0,37$; IC $95 \% 0,31-0,43 ; p<0,001)$, IMC inicial $(0,023$; IC $95 \%$ $0,004-0,042 ; \mathrm{p}=0,017)$, tratamiento inicial con insulina $(0,67$; IC 95\% 0,45-0,88; p<0,001) y necesidad de insulinización $(0,46 ;$ IC $95 \%$ 0,21-0,71; $<<0,001)$.

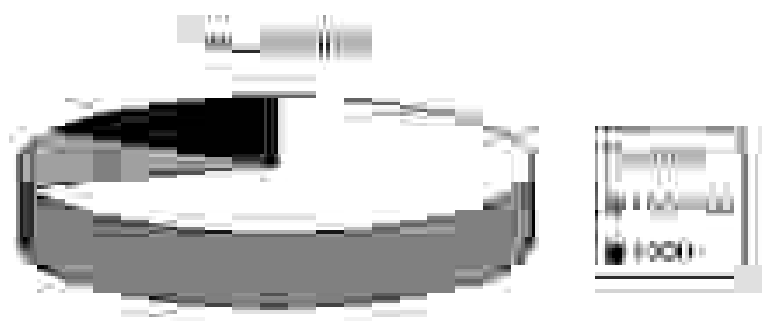

Hin
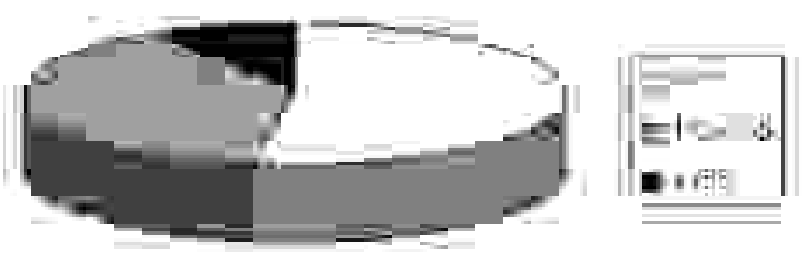

Fig. 2. Distribución de frecuencias de las cifras lipídicas 

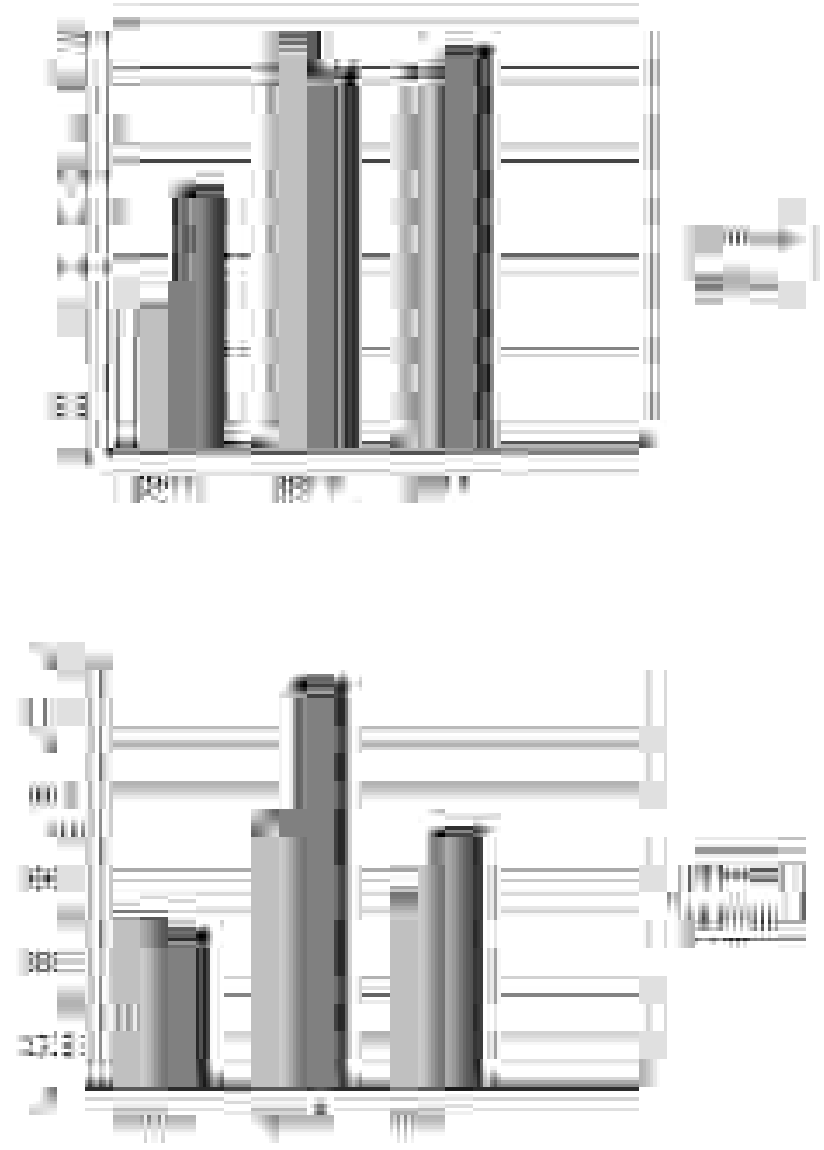

Fig. 3. Comparación de las cifras iniciales y finales de $\mathrm{HbAlc}$ y de IMC por subgrupos. ( $p=0,001$ para HbAlC en grupo $A ; p<0,001$ para IM C en grupos $B$ y C).

TABLA II

FACTORES PREDICTIVOS DE HBAIC M EDIA EN M ODELOS UNIVARIANTES

\begin{tabular}{lccc}
\hline & $\mathrm{b}$ & $\mathrm{IC} 95 \%$ & $\mathrm{R}$ cuadrado \\
\hline HbA1c inicial & 0,43 & $0,37-0,49$ & 0,38 \\
Tratamiento inicial con insulina & 0,68 & $0,45-0,9$ & 0,1 \\
Evolución & 0,025 & $0,011-0,041$ & 0,035 \\
Cambio de IMC & 0,026 & $0,011-0,041$ & 0,031 \\
Sexo varón & $-0,33$ & $(-0,57)-(-0,09)$ & 0,023 \\
Insulinización & 0,33 & $0,05-0,61$ & 0,017 \\
IMC inicial & 0,028 & $0,003-0,053$ & 0,015 \\
Edad & 0,05 & $(-0,08)-0,02$ & 0,002 \\
\hline
\end{tabular}

\section{DISCUSIÓN}

En el presente trabajo hemos comprobado como es más sencilla la consecución de los objetivos lipídicos y de tensión arterial que los objetivos glucémicos en pacientes con diabetes tipo 2. Así menos del $10 \%$ de nuestros pacientes tenían cifras de LDL colesterol superiores a $155 \mathrm{mg} / \mathrm{dl}$, consideradas por el European Diabetes Policy Group de alto riesgo. Menos favorable era el control de la tensión arterial, ya que casi un
$40 \%$ de los pacientes tenían cifras superiores a $140 \mathrm{mmHg}$ de sistólica y/o $85 \mathrm{mmHg}$ de diastólica; no obstante menos del $10 \%$ tenía cifras de tensión arterial sistólica superior a 160 $\mathrm{mmHg}$. Mención aparte merece el control glucémico, claramente inadecuado, ya que el $63 \%$ de los pacientes presentaba cifras superiores a 7,5\%, con una mediana de 7,9\%.

La dificultad de la consecución de los objetivos glucémicos está ampliamente constatada en la literatura. Así en una muestra de 733 diabéticos reclutados en el NHANES III (11) más de un $40 \%$ tenía cifras de $\mathrm{HbA} 1 \mathrm{c}>8 \%$ y un $15 \%$ cifras de HbA1c > 10\%; en esta población, además, un 33\% tenía cifras de tensión arterial superior a 140/90 y un $20 \%$ cifras de LDL superiores a $160 \mathrm{mg} / \mathrm{dl}$, a pesar de que el $90 \%$ de los pacientes había visitado al médico al menos 2 veces en el último año.

Específicamente pudimos demostrar una mejoría a lo largo del tiempo en las cifras de lípidos y de tensión arterial, pero no así en las cifras de HbA1c. Además, durante el seguimiento de 5 años, casi un $25 \%$ de los pacientes precisó insulinización. Este deterioro progresivo del control glucémico con el tiempo se constató en el UKPDS (12). En este trabajo, a lo largo de 6 años, un 53\% de pacientes tratados inicialmente con sulfonilureas precisó tratamiento adicional con insulina para mantener una glucemia basal $<108 \mathrm{mg} / \mathrm{dl}(13)$.

El objetivo durante el seguimiento del paciente diabético es mantener un promedio adecuado de $\mathrm{HbA} 1 \mathrm{c}$ para prevenir las complicaciones crónicas. Por ello intentamos determinar factores predictivos de este control medio.

Uno de los factores predictivos más importantes del deterioro del control glucémico durante el seguimiento es la pérdida de reserva insulínica (14-17). Por ello los datos de control glucémico de una cohorte deben valorarse en dependencia de la duración conocida de su enfermedad. Nuestros pacientes tenían una evolución mediana de 15 años, y en el momento de la visita final las dos terceras partes llevaban tratamiento con insulina, lo que traduce un deterioro importante de la reserva insulínica; no obstante no dispusimos de cifras de c-péptido para confirmar este punto. En regresión múltiple, dos parámetros indirectos de pérdida de reserva insulínica, como son el tratamiento inicial con insulina y la necesidad de insulinización durante el seguimiento, se revelaron como predictores significativos de la HbA1c media.

El sobrepeso está relacionado con la aparición y con la dificultad para controlar la diabetes tipo 2 (18). Además la mejoría del control glucémico frecuentemente se asocia a aumento de peso (19). En nuestro trabajo comprobamos cómo un IMC inicial elevado se asociaba a un incremento de la HbA1c durante el seguimiento. Además en pacientes en tratamiento con insulina o, sobre todo, insulinizados durante el seguimiento, había un incremento progresivo de peso corporal. Por ello es fundamental conseguir estrategias terapeúticas que consigan controlar el peso del paciente con diabetes (20).

Un tercer factor es el mal control metabólico inicial (18). En nuestro estudio pudimos comprobar la dificultad de conseguir un buen control glucémico en aquellos pacientes que en la visita inicial tenían una cifra de HbA1c elevada, y de hecho la $\mathrm{HbA} 1 \mathrm{c}$ inicial fue el principal factor predictivo del control metabólico durante el seguimiento. Al menos en nuestros pacientes, durante un seguimiento de 5 años, no detectamos un empeoramiento clínicamente importante de las cifras de HbA1c. Con una estrategia terapeútica semejante a la nuestra se ha descrito mejoría en el tiempo de las cifras de HbA1c, 
pero en este caso se trataba de pacientes con menor tiempo de evolución y el seguimiento se circunscribió a 2 años (21).

Podemos concluir diciendo que hemos constatado la dificultad de conseguir los objetivos de control glucémico recomendados por las sociedades internacionales en pacientes con diabetes tipo 2. Los principales factores asociados a elevación de la $\mathrm{HbA} 1 \mathrm{c}$ media durante un seguimiento de 5 años han sido los valores de la HbAlc y del IMC iniciales y la presencia inicial de tratamiento insulínico o la necesidad de insulinización.

\section{Bibliografía}

1. Tamayo Marco B, Faure Nogueras E, Roche Asensio MJ Rubio Calvo E, Sanchez Oriz E, Salvador Olivan JA. Prevalence of diabetes and impaired glucose tolerance in Aragón, Spain. Diabetes Care 1997; 20: 534-536.

2. The Diabetes Control and Complications Trial Research Group: The effect of intensive treatment of diabetes on the development and progression of long-term complications in insulin dependent diabetes mellitus. N Engl J Med 1993, 329: 977-986.

3. Ohkubo Y, Kishikawa H, Araki E, Miyata T, Isami S, Motoyoshi S et al: Intensive insulin therapy prevents the development of diabetic microvascular complications in Japanese patients with non insulin dependent diabetes mellitus: a randomized prospective 6-year study. Diab Res Clin Pract 1995; 28: 103-117.

4. U.K. Prospective Diabetes Study (UKPDS) Group: Intensive blood-glucose control with sulphonylureas or insulin compared with conventional treatment and risk of complications in patientes with type 2 diabetes (UKPDS 33). Lancet 1998; 352: 837-853.

5. Morrish NJ, Wang SL, Stevens LK, Fuller JH, Keen H and the WHO Multinational Study Group. Mortality and causes of death in the WHO multinational study of vascular disease in diabetes. Diabetologia 2001, 44(Supl 2): S14-S22.

6. Haffner SM, Alexander CM, Cook TJ, Boccuzzi SJ, Musliner TA, Pedersen TR et al. Reduced coronary events in simvastatin treated patients with coronary heart disease and diabetes or impaired fasting glucose levels. Arch Intern Med 1999; 159: 2661-2667.

7. UK Prospective Diabetes Study Group. Tight blood presure control and risk of macrovascular and microvascular complications in type 2 diabetes: UKPDS 38. BMJ 1998; 317: 703-713.

8. European Diabetes Policy Group. A desktop guide to type 2 diabetes mellitus. Diabet Med 1999; 16: 716-730.

9. American Diabetes Association. Standards of medical care for patients with diabetes mellitus. Diabetes Care 2002; 25 (Supl, 1): S33-S49.

10. The Expert Committee on the diagnosis and classification of Diabetes Mellitus. Report of the Expert Committee on the diagnosis and classification of Diabetes Mellitus. Diabetes Care 1997, 20: 11831197.
11. Harris MI. Health care and health status and outcomes for patients with type 2 diabetes. Diabetes Care 2000; 23: 754-758.

12. UK Prospective Diabetes Study Group. Overview of 6 years' therapy of type II diabetes: a progressive disease (UKPDS 16). Diabetes 1995; 44 1249-1258.

13. Wright A, Burden AC, Paisey RB, Cull CA, Holman RR and UK Prospective Diabetes Study Group. Sulfonylurea inadequacy: efficacy of addition of insulin over 6 years in patients with type 2 diabetes in the UK Prospective Diabetes Study (UKPDS 57). Diabetes Care 2002; 25: 330-336.

14. Blaum CS, Velez L, Hiss R, Halter JB. Charasteristics related to poor glycemic control in NIDDM patients in community practice. Diabetes Care 1997, 1: 7-11.

15. Nielsen NV, Tronier B. C-peptide and insulin secretion in diabetes mellitus treated with oral hypoglycaemic agents or diet alone. A 3 years epidemiological cohort study on the Island of Falster, Denmark. Diabetes Res 1987; 4: 135-139.

16. Niskanen L, Karjalainen J, Siitonen O, Uusitupa M. Metabolic evolution of type 2 diabetes: a 10 year follow-up from the time of diagnosis. J Intern Med 1994, 236: 263-270.

17. Chan WB, Chan JCN, Chow CC, Yeung VTF, So WY, Li JKY et al Glycaemic control in type 2 diabetes: the impact of body weight, b-cell function and patient education. Q J Med 2000; 93: 183-190.

18. Turner RC, Cull CA, Frighi V, Holman RR. Glycemic control with diet, sulfonylurea, metformin, or insulin in patients with type 2 diabetes mellitus. Progressive requirement for multiple therapies (UKPDS 49). JAMA 1999; 281: 2005-2012.

19. Makimattila S, Nikkila K, Yki-Jarvinen H. Causes of weight gain during insulin therapy with and without metformin in patients with type II diabetes mellitus. Diabetologia 1999, 42: 406-412.

20. Manzanares Errazu JM. Challenges in the treatment of patients with type 2 diabetes mellitus and obesity. Nutr Hosp 2002; 17: 1-6.

21. de Sonnaville JJ, Bouma M, Colly LP, Deville W, Wijkel D, Heine RJ Sustained good glycaemic control in NIDDM patients by implementation of structured care in general practice: 2 year follow-up study. Diabetologia 1997; 40: 1334-1440. 